\title{
Phase I study shows potential of TRC105 in mCRPC
}

TRC105 is well tolerated at a dose of $20 \mathrm{mg} / \mathrm{kg}$ every other week and shows antiangiogenic activity in patients with metastatic castration-resistant prostate cancer (mCRPC), say researchers.

Antiangiogenic agents have shown potential to reduce tumour growth and metastasis in CRPC. Various angiogenesis inhibitors (given either alone or in combination with chemotherapy) have been investigated in MCRPC but have not been shown to improve overall survival. TRC105 is a chimeric IgG1 monoclonal antibody that binds to human endoglin (CD105), a protein involved in normal vascular development that is highly expressed on the surface of vascular endothelial cells during tumour angiogenesis.

An earlier phase I study had shown that TRC105 administration had resulted in stable disease in 21 of 45 patients (47\%) with advanced cancer. Karzai et al. set out to determine the maximum tolerated dose and investigate the safety, pharmacokinetics, pharmacodynamics, and antitumour activity of TRC105 in patients with mCRPC.

The researchers treated 21 patients (divided into six cohorts) with escalating doses of intravenous TRC105 until unacceptable toxic effects occurred or disease progression was observed, up to a predetermined top dose of $20 \mathrm{mg} / \mathrm{kg}$ every 2 weeks. In total, 20 patients completed the 28-day dose-limiting toxicity evaluation period.

\section{Eight patients showed declines in PSA level, two of these $>50 \% 77$}

Patients received a median of four cycles of treatment (range 1-7 cycles). The maximum tolerated dose was the highest level studied $-20 \mathrm{mg} / \mathrm{kg}$ every 2 weeks. The most common adverse events were infusion-related reaction, headache, anaemia, epistaxis, fever, nausea, vomiting, bone pain and oral haemorrhage.
Of the 20 evaluable patients, seven had stable disease after two treatment cycles and three had stable disease after four treatment cycles. Eight patients showed declines in PSA level, two of these $>50 \%$. The researchers noted PSA declines across all dose levels without any clear dose responses. Karzai et al. also reported that increases in plasma VEGF occurred in patients with CD105 depletion after treatment with TRC106. They suggest that VEGF rising is a possible compensatory mechanism for TRC105-induced anti-angiogenic activity.

"Further studies of TRC105 in combination with AR [andogen receptor]targeted therapies, chemotherapy, VEGF inhibitors or immune checkpoint inhibitors in patients with mCRPC could be valuable in defining the clinical potential of TRC105 in prostate cancer," say the authors.

Rebecca Kelsey

Original article Karzai, F. H. et al. A phase I study of TRC105 anti-CD105 (endoglin) antibody in metastatic castrationresistant prostate cancer. BJU Int. doi:10.1111/bju.12986 